\title{
Thermal characterization of the multimeric bacteriophage endolysin PlyC
}

J. Todd Hoopes ${ }^{1,2 \S}$, Ryan D. Heselpoth ${ }^{1, \S}$, Frederick P. Schwarz ${ }^{1,2, \uparrow}$, and Daniel C. Nelson ${ }^{1,3, *}$

${ }^{1}$ Institute for Bioscience and Biotechnology Research, University of Maryland, Rockville, MD, 20850, USA.

${ }^{2}$ National Institute of Standards and Technology, Gaithersburg, MD, 20899, USA.

${ }^{3}$ Department of Veterinary Medicine, University of Maryland, College Park, MD, 20740, USA.

${ }^{\S}$ These authors contributed equally

†Deceased

*For correspondence: Daniel C. Nelson: E-mail: nelsond@umd.edu; Tel: +1-240-314-6249; Fax:

$+1-240-314-6225$

Keywords: PlyC, endolysin, antimicrobial, thermal stability, thermodynamic characterization, differential scanning calorimetry 


\section{Abstract}

Bacteriophage endolysins degrade the bacterial peptidoglycan and are considered enzymatic alternatives to small molecule antibiotics. In particular, the multimeric streptococcal endolysin PlyC has appealing antibacterial properties. However, a comprehensive thermal analysis of PlyC is lacking, which is necessary for evaluating long-term stability and downstream therapeutic potential. Biochemical and kinetic-based methods were used in combination with differential scanning calorimetry to investigate the structural, kinetic and thermodynamic stability of PlyC and its various subunits and domains. The PlyC holoenzyme structure is irreversibly compromised due to partial unfolding and aggregation at $46^{\circ} \mathrm{C}$. Unfolding of the catalytic subunit, PlyCA, instigates this event, resulting in the kinetic inactivation of the endolysin. In contrast to PlyCA, the PlyCB octamer (the cell wall binding domain) is thermostable, denaturing at $\sim 75^{\circ} \mathrm{C}$. Isolation of PlyCA or PlyCB alone altered their thermal properties. Contrary to the holoenzyme, PlyCA alone unfolds uncooperatively and is thermodynamically destabilized whereas the PlyCB octamer reversibly dissociates into monomers and forms an intermediate state at $74^{\circ} \mathrm{C}$ in phosphate buffered saline, with each subunit subsequently denaturing at $92^{\circ} \mathrm{C}$. Adding folded PlyCA to an intermediate state PlyCB, followed by cooling, allowed for in vitro reconstitution of the active holoenzyme.

\footnotetext{
Abbreviations: CBD, cell wall binding domain; CHAP, cysteine, histidine-dependent amidohydrolase/peptidase; DSC, differential scanning calorimetry; $\Delta H_{\text {cal }}$, calorimetric enthalpy; $\Delta H_{\mathrm{vH}}$, van't Hoff enthalpy; $T_{G}$, thermal transition temperature
} 


\section{Introduction}

The increasing global threat of bacterial resistance to the traditional antibiotics highlights the need for alternative antimicrobials $(1,2)$. One potential antimicrobial strategy involves the use of bacteriophage (or phage)-encoded endolysins $(3,4)$. These enzymes are primarily peptidoglycan (i.e., cell wall) hydrolases that cleave critical covalent bonds in the cell wall of phage-infected bacteria. Due to the elevated intracellular turgor pressure, the loss of peptidoglycan structural integrity causes hypotonic lysis and phage virion liberation. The antibacterial efficacy of extrinsically-applied recombinant endolysins toward sensitive bacteria has been validated both in vitro and in vivo $(5,6)$.

One example of an endolysin with established antibacterial activity of clinical interest is PlyC. This endolysin rapidly kills species of Streptococcus that cause both human and animal disease (7, 8). Interestingly, PlyC eradicates Streptococcus pyogenes biofilms and innately possesses the unique ability to translocate across epithelial cell membranes to kill intracellular streptococci (9, 10). In addition to its potent in vitro antibacterial properties, PlyC was highly effective in vivo when administered as either a prophylactic or therapeutic using a murine pharyngeal decolonization model (7).

The structure of PlyC has a novel multimeric composition (11). Contrary to the conventional protein architecture for endolysins that target Gram-positive bacteria, which generally consist of a single polypeptide with a conserved $\mathrm{N}$-terminal catalytic domain linked to a variable $\mathrm{C}$-terminal cell wall binding domain (CBD), the PlyC holoenzyme collectively consists of nine subunits. The CBD comprises eight identical PlyCB monomers that interact through protein-protein interactions 
to form an octameric ring structure (11). The PlyCB CBD was recently shown to bind with high affinity and avidity to a polyrhamnose moiety shared in the wall teichoic acids of sensitive streptococcal species $(12,13)$. The ninth subunit, PlyCA, is the catalytic subunit consisting of an N-terminal glycosyl hydrolase $(\mathrm{GyH})$ domain, a central helical docking domain, and a C-terminal cysteine, histidine-dependent amidohydrolase/peptidase (CHAP) domain. The GyH and CHAP domains act together synergistically to generate the robust bacteriolytic activity of PlyC, whereas the central helical docking domain of PlyCA interacts with the PlyCB CBD to form the holoenzyme structure (11).

Elucidating the thermal characteristics of a biologic system is necessary for assessing its long-term stability and therapeutic potential. Moreover, the Arrhenius equation describes the relationship between the rate of a chemical reaction and temperature, and thereby associates the thermal stability of a material in general, and a drug in particular, to its shelf-life expectancy. Accordingly, at a given temperature, the intrinsic stability of a protein directly correlates with the activation energy required for unfolding and, by extension, shelf-life $(14,15)$. With this in mind, we determined the thermal properties of PlyC. Due to the complexity of its multimeric structure, the PlyC holoenzyme, as well as individual subunits and domains, were thermally characterized using a combination of biological, functional and biophysical experimental approaches. Results from this analysis will ultimately aid in downstream developmental strategies for prolonging long-term stability of PlyC, such as identifying thermolabile structural components to target in subsequent protein engineering studies and excipient formulations. 


\section{Materials and methods}

\section{Bacterial strains, culture conditions, expression and purification}

S. pyogenes strain D471 was used for all experiments and routinely grown in Todd Hewitt broth supplemented with $1 \%(\mathrm{w} / \mathrm{v})$ yeast extract as previously described $(7,16)$. Escherichia coli strains used for protein expression were grown at $37^{\circ} \mathrm{C}$ in $4 \mathrm{~L}$ baffled flasks containing $1.5 \mathrm{~L}$ of LuriaBertani and ampicillin (100 $\mu \mathrm{g} / \mathrm{ml}$ when needed) with shaking at 190 revolutions per minute. Recombinant PlyC, PlyCA, PlyCB and CHAP were expressed and purified as previously described (11).

\section{Thermal denaturation of PlyC}

PlyC aliquots at $1 \mathrm{mg} / \mathrm{ml}$ were incubated in phosphate buffered saline (PBS), $\mathrm{pH} \mathrm{7.2,} \mathrm{using}$ EchoTherm ${ }^{\mathrm{TM}}$ heat blocks (Torrey Pines Scientific) at $37,42,50,55,60$ or $65^{\circ} \mathrm{C}$ for $30 \mathrm{~min}$. The samples were then removed and immediately cooled on ice. The structural integrity of the holoenzyme was assessed using native polyacrylamide gel electrophoresis (PAGE). Sodium dodecyl sulfate polyacrylamide gel electrophoresis (SDS-PAGE) was used as a control to confirm the presence of both the PlyCA and PlyCB subunits at each temperature. Once the temperature range for thermal denaturation was narrowed, the experiment was repeated, but at temperatures of $42,43,44,45,46,47$ or $48^{\circ} \mathrm{C}$.

\section{Kinetic inactivation assay}

Kinetic stability assays were performed by initially heating purified PlyC in PBS at $1 \mathrm{mg} / \mathrm{ml}$ in EchoTherm ${ }^{\mathrm{TM}}$ heat blocks to the same temperatures used above for the thermal denaturation gels for $10 \mathrm{~min}$, followed by cooling on ice. Next, a turbidity reduction assay was used to measure 
residual bacteriolytic activity. For this, an overnight culture of $S$. pyogenes was harvested, washed and resuspended in PBS to an optical density at $600 \mathrm{~nm}\left(\mathrm{OD}_{600 \mathrm{~nm}}\right)$ of 1.0. Equal volumes $(100 \mu 1$ each) of the heated PlyC samples and S. pyogenes were mixed into individual wells of a 96-well flat-bottomed microtiter plate. Using a SpectraMax 190 spectrophotometer (Molecular Devices), the $\mathrm{OD}_{600 \mathrm{~nm}}$ was measured every $10 \mathrm{sec}$ for a total of $5 \mathrm{~min}$ at room temperature. The final $\mathrm{OD}_{600 \mathrm{~nm}}$ was recorded and the data was normalized to the activity of PlyC without heat treatment.

\section{Differential scanning calorimetry}

Differential scanning calorimetry (DSC) measurements were performed using a VP-DSC Microcalorimeter (MicroCal Inc.) under a static, oxidative atmosphere. The sample and reference vessels had an operational volume of $0.511 \mathrm{ml}$. Prior to sample analysis, PBS, pH 7.4, or $20 \mathrm{mM}$ phosphate buffer, $\mathrm{pH}$ 7.4, was initially added to both the sample and reference vessels and subsequently subjected to two heating/cooling cycles from 15 to $105^{\circ} \mathrm{C}$ and 105 to $15^{\circ} \mathrm{C}$ using scan rates of either 15 or $60 \mathrm{~K} / \mathrm{h}$. The result from the second heating/cooling cycle was utilized as the base line. Next, protein samples at various concentrations were independently injected into the sample vessel and subjected to multiple heating/cooling cycles. After subtraction of the baseline from the sample scan, the resulting raw data of the differential power as a function of time was divided by the scan rate to convert the data into heat capacity versus temperature. Utilizing the EXAM program (17), two-state thermal transition models were fitted to the heat capacity versus temperature data to calculate the van't Hoff enthalpy $\left(\Delta H_{\mathrm{VH}}\right)$ from the shape of the transition peak. EXAM was also used to determine $T_{\mathrm{G}}$ (the temperature at the thermal transition midpoint) and calorimetric enthalpy $\left(\Delta H_{\text {cal }}\right.$; the area under the transition peak divided by the moles of protein in the sample cell) values. 


\section{PlyCB cross-linking}

At $1 \mathrm{mg} / \mathrm{ml}, 100 \mu \mathrm{l}$ aliquots of the PlyCB CBD in PBS were heated for $10 \mathrm{~min}$ in a gradient thermocycler (MyCycler, BioRad) at temperatures ranging from 25 to $90^{\circ} \mathrm{C}$. Following the instructions provided by the manufacturer, the amine groups of the heated PlyCB samples were crosslinked with bis(sulfosuccinimidyl) suberate (ThermoFisher Scientific) for $10 \mathrm{~min}$. After the reaction was quenched with $10 \mu \mathrm{l}$ of $1 \mathrm{M}$ Tris, $\mathrm{pH}$ 7.4, the heated samples were immediately placed on ice for $10 \mathrm{~min}$ and ultimately resolved via SDS-PAGE.

\section{Bright field and fluorescence microscopy}

The organic dye, AlexaFluor ${ }^{\mathrm{TM}}-555$ (ThermoFisher Scientific), was conjugated to PlyCB according to the manufacturer's instructions. Aliquots consisting of $100 \mu 1$ of the conjugated protein at $1 \mathrm{mg} / \mathrm{ml}$ were incubated from 4 to $100^{\circ} \mathrm{C}$ for $30 \mathrm{~min}$ in $\mathrm{PBS}$ using an EchoTherm ${ }^{\mathrm{TM}}$ refrigerated heat block and then immediately cooled on ice. Next, the protein samples (10 $\mu 1$ each) were incubated with $S$. pyogenes in PBS for $10 \mathrm{~min}$ at room temperature. The labeled cells were washed with PBS and viewed on a Nikon Eclipse 80i fluorescent microscope equipped with a Retiga 2000R camera using a Cy3 filter for fluorescence imaging.

\section{Thermal regeneration of native PlyC from its constituent subunits}

At a concentration of $15.6 \mu \mathrm{M}(1 \mathrm{mg} / \mathrm{ml}), 500 \mu \mathrm{l}$ of the PlyCB CBD in PBS was placed in an EchoTherm ${ }^{\mathrm{TM}}$ heat block and heated to $75^{\circ} \mathrm{C}$. Next, $500 \mu \mathrm{l}$ of $19.5 \mu \mathrm{M}(1 \mathrm{mg} / \mathrm{ml})$ ice-cold PlyCA was rapidly added via a syringe to the heated PlyCB sample. Alternately, an identical experiment was carried out with both PlyCA and PlyCB protein samples at room temperature. Immediately 
after mixing, all samples were placed in an ice bath and allowed to cool for 5 min. Samples were tested for residual lytic activity against $S$. pyogenes using a turbidity reduction assay as previously described (see Kinetic inactivation assay). Relative lytic activity was normalized to that of wildtype PlyC, and equal molar concentrations of isolated PlyCA or PlyCB CBD were used as controls. To further establish that the PlyC holoenzyme had been reconstituted by mixing heated PlyCB with cold PlyCA, the remaining sample was centrifuged at $16,000 \times \mathrm{g}$ for 10 min to remove any precipitate and loaded onto a HiPrep 16/60 Sephacryl S-200 HR size-exclusion column (GE Healthcare) pre-equilibrated in PBS. An elution peak corresponding to native PlyC was collected and subsequently analyzed by SDS-PAGE.

\section{Results}

\section{Thermal denaturation of PlyC}

The thermal stability of the PlyC holoenzyme structure was initially examined by incubating equal molar concentrations of the purified endolysin at temperatures ranging from 37 to $65^{\circ} \mathrm{C}$ for 30 minutes, followed by rapid cooling and visual inspection using native PAGE (Fig. 1). Results from the initial electrophoretic analysis suggest the structural conformation of PlyC is compromised between 42 and $50^{\circ} \mathrm{C}$, as evidenced by the inability of the aggregated sample to enter a native polyacrylamide gel (Fig. 1, left). When samples at all temperatures were analyzed on an SDSPAGE gel, the PlyCA and PlyCB subunits were observed at relatively equal concentrations (data not shown), thus proving the absence of bands on native PAGE for PlyC treated at $\geq 50^{\circ} \mathrm{C}$ can be directly attributed to protein unfolding and aggregation. Next, $\mathrm{PlyC}$ was heated from 42 to $48^{\circ} \mathrm{C}$ in one degree increments in order to determine the precise temperature the holoenzyme loses structural integrity. Native PAGE revealed PlyC loses electrophoretic mobility at $46^{\circ} \mathrm{C}$, as a result 
of thermally-induced aggregation (Fig. 1, right). These observations directly correlate with a temperature-dependent loss of enzymatic activity toward S. pyogenes (Fig. 1, bottom).

\section{Thermodynamic characterization of PlyC}

DSC was performed to investigate the thermodynamic properties of the PlyC holoenzyme, as well as various isolated subunits and domains of the enzyme. Each protein sample investigated was purified to near homogeneity (Fig. 2). For this thermodynamic analysis, each thermal transition detected was fit with either an $\mathrm{A} \leftrightarrow \mathrm{B}$ or $\mathrm{A}_{2} \leftrightarrow 2 \mathrm{~B}$ two-state transition models specific to the heat capacity versus temperature data. The resulting $T_{G}$ and enthalpy values obtained for each symmetrical or asymmetrical thermal denaturation transition corresponds to the two-state transition model that fit best. Transition properties that are considered to be scan rate independent, which can be concluded when the $T_{G}$ values with twice their uncertainties are within $3^{\circ} \mathrm{C}$ and the enthalpies are within twice their uncertainties, are capable of being analyzed by thermodynamic two-state transition models (18).

First, PlyCA was thermodynamically characterized at 1 to $2 \mathrm{mg} / \mathrm{ml}$ in PBS using scan rates of 15 and $60 \mathrm{~K} / \mathrm{h}$ (Table 1). As an example, the DSC thermogram of PlyCA is shown in Fig. 3A. DSC scans of PlyCA were fit with two $\mathrm{A} \leftrightarrow \mathrm{B}$ two-state transitions and thus fulfills a three-state thermal transition model. At $15 \mathrm{~K} / \mathrm{h}$, thermal transitions for PlyCA are observed at $41.4^{\circ} \mathrm{C}$ and $44.6^{\circ} \mathrm{C}$, with respective $\Delta H_{\mathrm{vH}}$ values of $845 \mathrm{~kJ} / \mathrm{mol}$ and $674 \mathrm{~kJ} / \mathrm{mol}$. At $60 \mathrm{~K} / \mathrm{h}$, PlyCA thermal transitions are observed at $42.0^{\circ} \mathrm{C}$ and $48.6^{\circ} \mathrm{C}$, with $\Delta H_{\mathrm{vH}}$ values of $302 \mathrm{~kJ} / \mathrm{mol}$ and $545 \mathrm{~kJ} / \mathrm{mol}$, respectively. The sum of the van't Hoff enthalpies at each scan rate equals the calorimetric enthalpy, thereby indicating the thermodynamic domains of PlyCA unfold independently at these temperatures. 
Changes in heat capacity were not observed after cooling and rescanning the heated samples, suggesting the unfolding of PlyCA is irreversible following thermal denaturation.

Next, the C-terminal CHAP domain of PlyCA was thermodynamically investigated at 1 to $2 \mathrm{mg} / \mathrm{ml}$ in PBS using scan rates of 15 and $60 \mathrm{~K} / \mathrm{h}$ (Table 2). Similar to PlyCA, the resulting thermograms of the CHAP domain were each fit with two $\mathrm{A} \leftrightarrow \mathrm{B}$ two-state transitions. At $15 \mathrm{~K} / \mathrm{h}$, the CHAP domain displays thermal transitions at 38.5 and $44.3^{\circ} \mathrm{C}$, with respective $\Delta H_{\mathrm{vH}}$ values of 464 and $583 \mathrm{~kJ} / \mathrm{mol}$. When using a scan rate of $60 \mathrm{~K} / \mathrm{h}$, thermal transitions were observed at 39.1 and $46.1^{\circ} \mathrm{C}$, with corresponding $\Delta H_{\mathrm{vH}}$ values of 430 and $554 \mathrm{~kJ} / \mathrm{mol}$. The ratios of the calorimetric enthalpy to the van't Hoff enthalpies were close to 0.5 , indicating the CHAP domain unfolds as a single cooperative unit. Repeating DSC scans with previously heated protein samples confirmed the unfolding of the CHAP domain is an irreversible thermodynamic process.

The thermodynamic properties of the octameric PlyCB CBD were elucidated at either $1 \mathrm{mg} / \mathrm{ml}$ in PBS (Table 3) or 1 to $2 \mathrm{mg} / \mathrm{ml}$ in phosphate buffer, $\mathrm{pH} 7.4$ (Table 4). An example of a thermogram specific to the PlyCB CBD is shown in Fig. 3B. Results from the DSC scans indicate the PlyCB CBD fulfills a three-state thermal transition model. The lower temperature thermal transition was fit with a two-state $\mathrm{A}_{2} \leftrightarrow 2 \mathrm{~B}$ model. This transition occurs either at $73.7^{\circ} \mathrm{C}$ with a $\Delta H_{\mathrm{vH}}$ value of $1265 \mathrm{~kJ} / \mathrm{mol}$ in PBS, or at $70.4^{\circ} \mathrm{C}$ with a $\Delta H_{\mathrm{vH}}$ value of $1160 \mathrm{~kJ} / \mathrm{mol}$ in phosphate buffer. The higher temperature thermal transition, which was fit with a two-state $\mathrm{A} \leftrightarrow \mathrm{B}$ model, was observed either at $91.8^{\circ} \mathrm{C}$ with a $\Delta H_{\mathrm{vH}}$ value of $368 \mathrm{~kJ} / \mathrm{mol}$ in $\mathrm{PBS}$, or at $83.5^{\circ} \mathrm{C}$ with a $\Delta H_{\mathrm{vH}}$ value of 373 $\mathrm{kJ} / \mathrm{mol}$ in phosphate buffer. The ratio of the transition enthalpy to the van't Hoff enthalpy for the $\mathrm{A}_{2} \leftrightarrow 2 \mathrm{~B}$ transition was nearly 2 , indicating the PlyCB octamer dissociates into an intermediate 
state. This intermediate state is followed by denaturation of the PlyCB subunit at the higher temperature transition of $91.8^{\circ} \mathrm{C}(\mathrm{PBS})$ or $83.5^{\circ} \mathrm{C}$ (phosphate buffer). Thus, the dissociation of the PlyCB octamer, followed by the thermal denaturation of the individual PlyCB monomers, can be summarized as $\mathrm{A}_{2} \leftrightarrow 2 \mathrm{I} \leftrightarrow 2 \mathrm{~B}$, were $\mathrm{I}$ is the intermediate state. Results from rescanning previously heated PlyCB samples indicate both thermal transitions are reversible. An example of a typical thermogram obtained from these experiments is depicted in Fig. 3C. However, the $\mathrm{A}_{2} \leftrightarrow$ 2B transition appears at a lower temperature due to the significant reduction of folded PlyCB subunits upon rescanning, which in turn decreases the amount of protein that re-associates in the native octameric state. Additionally, the van't Hoff enthalpy specific to the $\mathrm{A}_{2} \leftrightarrow 2 \mathrm{~B}$ transition of PlyCB was lower when rescanning the protein samples, perhaps due to the steep dependence of the van't Hoff enthalpies on temperature.

DSC scans of the PlyC holoenzyme were performed at concentrations ranging from 0.81 to 3.82 $\mathrm{mg} / \mathrm{ml}$ in PBS using scan rates of 15 and $60 \mathrm{~K} / \mathrm{h}$ (Table 5). An example of a DSC thermogram of PlyC is shown in Fig. 3D. The complete thermal denaturation of PlyC satisfies a four-state thermal transition model. At $15 \mathrm{~K} / \mathrm{h}$, the first two thermal transitions observed at 44.5 and $48.4^{\circ} \mathrm{C}$, which had respective $\Delta H_{\mathrm{vH}}$ values of 677 and $862 \mathrm{~kJ} / \mathrm{mol}$, were fit with two $\mathrm{A} \leftrightarrow \mathrm{B}$ two-state transitions. The third thermal transition at $73.7^{\circ} \mathrm{C}$, which displayed a $\Delta H_{\mathrm{vH}}$ value of $1238 \mathrm{~kJ} / \mathrm{mol}$, was fit with a $\mathrm{A}_{2} \leftrightarrow 2 \mathrm{~B}$ two-state model. When using a scan rate of $60 \mathrm{~K} / \mathrm{h}$, the initial two thermal transitions are seen at 46.2 and $50.6^{\circ} \mathrm{C}$, with respective $\Delta H_{\mathrm{vH}}$ values of 472 and $637 \mathrm{~kJ} / \mathrm{mol}$, were fit with two $\mathrm{A} \leftrightarrow \mathrm{B}$ two-state transitions. The third thermal transition at $75.0^{\circ} \mathrm{C}$, which displayed a $\Delta H_{\mathrm{vH}}$ value of $1082 \mathrm{~kJ} / \mathrm{mol}$, was fit with a $\mathrm{A}_{2} \leftrightarrow 2 \mathrm{~B}$ two-state model. The lower temperature thermal transitions of PlyC at both scan rates correlate to the thermal denaturation of PlyCA. The ratio of 
the calorimetric enthalpy to the van't Hoff enthalpies is nearly 0.5 , suggesting the GyH and CHAP domains of PlyCA are unfolding cooperatively. The higher temperature thermal transition is specific to the thermal denaturation of the PlyCB CBD. This thermal transition equates to the $\mathrm{A}_{2}$ $\leftrightarrow 2 \mathrm{~B}$ transition displayed when scanning the PlyCB CBD alone. The $\mathrm{A} \leftrightarrow \mathrm{B}$ transition at $91.8^{\circ} \mathrm{C}$ observed with PlyCB alone is not observed when scanning the holoenzyme. Similar to DSC experiments using PlyCA and PlyCB CBD alone, cooling and rescanning previously heated PlyC samples revealed the respective irreversible and reversible folding characteristics of PlyCA and the PlyCB CBD. Only a small subpopulation of PlyCB subunits refolded, resulting in a decreased thermal transition temperature and van't Hoff enthalpy when compared to the initial DSC scan.

\section{PlyCB octamer structural integrity and kinetic stability as a function of temperature}

The DSC results for the isolated PlyCB CBD in PBS dictate the octamer reversibly dissociates into an intermediate state at $73.7^{\circ} \mathrm{C}$, which is then followed by the individual subunits thermally denaturing at $91.8^{\circ} \mathrm{C}$ (Table 3). To further expand on these findings, the PlyCB CBD was heated in PBS to temperatures ranging from 65 to $90^{\circ} \mathrm{C}$, followed by chemical cross-linking in order to qualitatively assess the structural integrity of the octamer via SDS-PAGE. At temperatures $\leq$ $74.4^{\circ} \mathrm{C}$, monomers, dimers, trimers and progressively higher ordered species of PlyCB were observed (Fig. 4A). In contrast, at temperatures exceeding $74.4^{\circ} \mathrm{C}$, there is a distinct change in the manner the PlyCB subunits interact, resulting in only monomers and dimers of PlyCB being observed. Consistent with the DSC findings, these results indicate the PlyCB octamer experiences a thermally-induced change in quaternary structure at temperatures $\geq 75^{\circ} \mathrm{C}$. 
When incubating the PlyCB CBD at temperatures below its thermal denaturation temperature, the reversibility of its intermediate state implies the octamer should be able to re-associate upon cooling and thus retain its ability to bind target streptococci. To this end, fluorescently-labeled PlyCB CBD was incubated in PBS at temperatures ranging from 4 to $100^{\circ} \mathrm{C}$, cooled and then assayed for binding to $S$. pyogenes using fluorescence microscopy (Fig. 4B). At temperatures $\leq$ $90^{\circ} \mathrm{C}$, the PlyCB CBD is capable of either maintaining or refolding into its octameric structure in order to bind the streptococcal surface. Alternatively, incubation at $100^{\circ} \mathrm{C}$ inhibits $\mathrm{PlyCB}$ functionality due to the thermal denaturation of its subunits. This is functional evidence the intermediate state of PlyCB is reversible.

\section{Refolding of the PlyC holoenzyme}

With the dissociation temperature and the reversibility of the intermediate state of PlyCB being experimentally validated, the folding dynamics of the PlyC holoenzyme were investigated next as a function of the PlyCB quaternary structure. A series of functional assays were performed to determine if PlyC can be reconstituted by mixing PlyCA with either PlyCB in its octameric state or with individual PlyCB subunits concurrently oligomerizing. For the initial set of control experiments, equal molar concentrations of PlyC, PlyCA or PlyCB CBD were added to S. pyogenes and the bacteriolytic activity was subsequently assessed using turbidity reduction assays (Fig. 5). Data was normalized to the lytic activity displayed by native PlyC. As expected, in the absence of the PlyCA catalytic subunit, the PlyCB CBD displays no lytic activity towards $S$. pyogenes. Furthermore, without the PlyCB CBD to direct the catalytic subunit to its peptidoglycan substrate, PlyCA alone exhibited $<1 \%$ in relative activity compared to the holoenzyme. Next, using a molar ratio of approximately 1:1, PlyCA was mixed with the PlyCB CBD at room temperature. The 
bacteriolytic activity displayed by the resulting mixture was equal to that of PlyCA alone, suggesting the structure of the PlyC holoenzyme was not regenerated. With this understanding, a near equal molar concentration of ice-cold PlyCA was rapidly mixed with PlyCB that had been heated to $75^{\circ} \mathrm{C}$, and the mixture was then rapidly cooled. In this scenario, the PlyCB octamer dissociates into an intermediate state when heated and then promptly re-associates when PlyCA is added and the mixture is cooled. When added to $S$. pyogenes, the reconstituted enzyme retained $35 \%$ of the relative bacteriolytic activity of native PlyC. Results from gel filtration indicated the aforementioned reconstituted enzyme had a molecular mass of $\sim 120 \mathrm{kDa}$ (data not shown), which equates to the mass of the $\mathrm{PlyC}$ holoenzyme. This is both functional and biochemical evidence that PlyCA is interacting with the PlyCB octamer to regenerate the PlyC holoenzyme structure.

\section{Discussion}

There is currently a deficiency in studies directed towards obtaining a thorough understanding of the thermal characteristics of bacteriophage endolysins. Results from such analyses would allow for a more complete and accurate assessment of the therapeutic potential of the biologic, as well as provide insightful information for downstream development. Previous studies evaluating the thermal properties of PlyC revealed the enzyme has modest kinetic stability (19-21). This finding seemed to be attributed to the thermosusceptiblility of PlyCA. To further confirm this observation, an extensive thermal characterization of PlyC was performed. When isolating each component, DSC analysis in PBS showed that the thermodynamic stability of PlyCA $\left(T_{G} \sim 42^{\circ} \mathrm{C}\right.$; Fig. $3 \mathrm{~A}$ and Table 1) is about $32^{\circ} \mathrm{C}$ lower than that of the PlyCB CBD $\left(T_{G} \sim 74^{\circ} \mathrm{C}\right.$; Table 3$)$. Using DSC to analyze the thermodynamics of the PlyC holoenzyme revealed the PlyCA subunit unfolds initially, displaying two cooperative thermal transitions between approximately $45^{\circ} \mathrm{C}$ and $50^{\circ} \mathrm{C}$ (Fig. 3D 
and Table 5). As confirmed by both DSC and functional assays, PlyCA unfolding is irreversible, which consequently results in kinetic inactivation of the enzyme at $46^{\circ} \mathrm{C}$ in PBS (Fig. 1, bottom). Following PlyCA unfolding, the PlyCB CBD thermally denatures at $\sim 74^{\circ} \mathrm{C}$ (Fig. 3D and Table 5).

There are notable differences in the thermal transition properties of both PlyCA and the PlyCB CBD when each component is analyzed either alone or complexed as a holoenzyme. For example, the thermodynamic stability of PlyCA is enhanced by about $4^{\circ} \mathrm{C}$ when complexed with the PlyCB octamer (compare Tables 1 and 5). Moreover, the ratios of the calorimetric enthalpy to the sum of the van't Hoff enthalpies specific to the two PlyCA thermal transitions are close to 0.5 and 1 in the PlyC and PlyCA only samples, respectively. These ratios indicate the GyH and CHAP domains of PlyCA can unfold independently when the catalytic subunit is uncomplexed, or as a single cooperative unit when PlyCA is anchored to the PlyCB CBD in the PlyC holoenzyme. These observations signify the GyH and CHAP domains interact strongly when PlyCA is bound to the PlyCB CBD, which is consistent with the synergistic activities noted between these two domains in the holoenzyme (11). Like PlyCA, the thermal transition properties of the PlyCB CBD vary depending on whether the octameric binding domain is analyzed alone or complexed with the catalytic subunit. DSC scans of the PlyCB CBD alone in PBS shows two uncooperative thermal transitions at $74^{\circ} \mathrm{C}$ (octamer dissociation) and $92^{\circ} \mathrm{C}$ (PlyCB monomer unfolding) (Table 3). Thermal cross-linking experiments supported these results, with the PlyCB octamer appearing to dissociate at temperatures $\geq 75^{\circ} \mathrm{C}$ (Fig. 4A). Additionally, heating/cooling experiments coupled with fluorescence microscopy confirmed that PlyCB can maintain or refold into its octameric structure to bind $S$. pyogenes until heated to $100^{\circ} \mathrm{C}$ (Fig. 4B). While the PlyCB $\mathrm{A}_{2} \leftrightarrow 2 \mathrm{~B}$ transition is maintained during DSC scans of PlyC, the higher $\mathrm{A} \leftrightarrow \mathrm{B}$ transition at $92^{\circ} \mathrm{C}$ is absent (Table 5). 
This result suggests PlyCB unfolding in the context of the holoenzyme does not proceed through an intermediate state. Instead, it appears the PlyCB monomers simultaneously unfold as the octamer dissociates.

When analyzing the calorimetric data for the PlyCB CBD in PBS (Table 3) and phosphate buffer (Table 4), it is evident that buffer composition has a significant effect on stability. Compared to phosphate buffer, the $T_{\mathrm{G}}$ for the $\mathrm{A}_{2} \leftrightarrow 2 \mathrm{~B}$ and $\mathrm{A} \leftrightarrow \mathrm{B}$ thermal transitions of the PlyCB CBD was increased by a respective 3.3 and $8.3^{\circ} \mathrm{C}$ in PBS. The thermodynamic stability of both the octamer and the individual PlyCB monomers appear to be enhanced by the presence of salt. As seen with other proteins (e.g., (22-24)), optimizing surface charge-charge networks by displacing water with electrostatic interactions between salts and proteins can improve stability (25). For the PlyCB $\mathrm{CBD}$, a direct relationship between salt concentration and protein stability can be assayed by performing future DSC experiments in phosphate buffer supplemented with varying concentrations of $\mathrm{NaCl}$.

The crystal structure of the uncomplexed PlyCB octamer shows disordered electron density for the eight N-terminal residues of each PlyCB monomer (11), presumably due to flexibility of this region. However, in the crystal structure of the PlyC holoenzyme, these same N-terminal amino acids form at least a four-stranded parallel $\beta$-sheet that creates a platform upon which the PlyCA central docking domain interacts via an antiparallel bundle of three $\alpha$-helices. These data suggest PlyCA and PlyCB fold together at the time of translation. Indeed, co-expression of PlyCA and PlyCB on separate plasmids allows for the reconstitution of a functional PlyC holoenzyme structure (26), yet mixing pre-formed PlyCA and PlyCB CBD at room temperature does not yield 
the active holoenzyme (Fig. 5). We therefore sought to elucidate whether the pre-folded state of the PlyCB CBD could be simulated based on its unique thermodynamic properties in order to study the folding dynamics of PlyC. In its uncomplexed form, the thermally-induced reversible folding of the PlyCB octamer allowed for the in vitro reconstitution of the PlyC holoenzyme. This was accomplished by adding ice-cold PlyCA to PlyCB in a heated intermediate state, and subsequently cooling the mixture on ice (Fig. 5). These findings indicate that the holoenzyme forms by PlyCA complexing with individual PlyCB subunits as they are simultaneously selfassociating into the octameric ring structure.

There is preliminary evidence to support that the CHAP domain of PlyCA is the most thermosusceptible structural component of PlyC. The isolated $\mathrm{CHAP}$ domain unfolds at $39^{\circ} \mathrm{C}$ (Table 2), which is a $3^{\circ} \mathrm{C}$ decrease from that of the isolated full-length PlyCA subunit (Table 1). Additional PlyC constructs will need to be thermodynamically investigated to definitively prove whether the CHAP domain is indeed the least thermostable component of the holoenzyme. Such constructs would include PlyC $\triangle \mathrm{CHAP}$ (i.e., PlyC holoenzyme without the CHAP domain of PlyCA), the isolated GyH domain of PlyCA, PlyC $\Delta \mathrm{GyH}$ (i.e., PlyC holoenzyme without the GyH domain of PlyCA) and PlyC $\Delta \Delta$ (i.e., the PlyCB CBD complexed with the helical docking domain of PlyCA only).

The overall findings from this study indicate the PlyCB CBD is highly thermostable, while the PlyCA subunit is relatively thermolabile. This general understanding will aid in formulating future engineering strategies for enhancing the thermal stability of PlyC. For example, PlyCA can be subjected to an optimized directed evolution-based methodology specific to increasing the thermal 
stability of bacteriolytic enzymes; this method was validated using PlyC (21). Alternatively, the PlyC crystal structure allows for structure-guided engineering approaches to be employed. Structural characteristics conserved in thermophilic proteins can be incorporated into PlyCA using rationale-based site-directed mutagenesis, such as substituting uncharged polar amino acids with charged residues to increase surface charge-charge interactions, shortening loop and turn structures, reducing the occurrence of thermolabile residues, and decreasing the size and amount of hydrophobic core cavities (27). As shown in previous studies, intra- and inter-domain disulfide bonds can be introduced to decrease the entropy associated with the folded or unfolded state of the protein, thereby increasing stability $(28,29)$. Computational methods utilizing in silico protein folding algorithms can also be used to estimate free energy values of folding, with a goal of predicting point mutations that are thermally advantageous to PlyCA. This strategy has already been proven useful for increasing the thermal stability of endolysins, specifically PlyC. Applying the Rosetta and FoldX protein folding algorithms to the CHAP domain of PlyCA allowed for the identification of a single point mutation that yielded a 16-fold increase in kinetic stability when compared to wild-type (20). Domain swapping is another engineering approach that can be used to increase the thermal stability of PlyC. For example, due to its modular structure, the CHAP domain of PlyCA can be substituted with a catalytic domain originating from an intrinsically thermostable peptidoglycan hydrolase. It is worth noting, however, that this type of engineering approach may not be optimal for a complex endolysin like PlyC, as the synergism shared between the GyH and CHAP domains may be disrupted and generate significant activity defects.

\section{Conclusions}


The thermolabile nature of the bacteriolytic mechanism of PlyC has been understood since the early 1970s (19). An in-depth thermal characterization has been lacking to identify the structural component(s) of the holoenzyme that is/are responsible for the modest stability of the endolysin. Results from our studies revealed the PlyCB CBD of PlyC is highly thermostable, whereas the PlyCA catalytic subunit is reasonably thermosusceptible. We speculate that the poor intrinsic stability of PlyCA could be due to its C-terminal CHAP domain, which was shown to be the most thermodynamically unstable PlyC-related construct analyzed. However, this result is preliminary, as additional PlyC constructs focusing on the GyH and helical docking domains of PlyCA need to be designed and studied using DSC.

There were significant differences in the thermal transition properties of the PlyC holoenzyme compared to its isolated catalytic subunit and CBD. For example, the GyH and CHAP domains of PlyCA appear to unfold independently when the catalytic subunit is isolated. When PlyCA is complexed to the PlyCB octamer, however, the GyH and CHAP domains interact strongly and unfold cooperatively. The thermodynamic stability of the PlyCA subunit was also enhanced when bound to the PlyCB CBD. In the context of the PlyC holoenzyme, the PlyCB CBD undergoes a single thermal transition corresponding to the simultaneous dissociation of the octamer, as well as each subunit denaturing. When isolated, the PlyCB octamer dissociates into a reversible intermediate state, which is then followed by a higher temperature thermal transition relating to each subunit denaturing. Adding ice-cold PlyCA to the intermediate state of PlyCB, followed by rapid cooling, resulted in the reconstitution of an enzymatically-active holoenzyme structure. This observation could aid in understanding the in vivo folding dynamics of this complex, multimeric endolysin. 


\section{Conflict of interest}

DCN is co-inventor of patents related to the PlyC endolysin.

\section{Acknowledgements}

This work was supported by USDA/NIFA Award \# 2017-05698. We would like to thank the National Institute of Standards and Technology for sharing their VP-DSC Microcalorimeter. This manuscript is dedicated to our late co-author, Frederick P. Schwarz.

\section{Author contributions}

JTH: Conceptualization, Data curation, Formal analysis, Writing - original draft. RDH: Formal analysis, Validation, Writing - review and editing. FPS: Conceptualization, Data curation, Formal analysis, Writing - original draft. DCN: Project administration, Funding acquisition, Formal analysis, Writing - review and editing. 


\section{References}

1. CDC. (2019) Antibiotic resistance threats in the United States, 2019. U.S. Department of Health and Human Services, Atlanta, GA

2. Czaplewski, L., Bax, R., Clokie, M., Dawson, M., Fairhead, H., Fischetti, V. A., Foster, S., Gilmore, B. F., Hancock, R. E., Harper, D., Henderson, I. R., Hilpert, K., Jones, B. V., Kadioglu, A., Knowles, D., Olafsdottir, S., Payne, D., Projan, S., Shaunak, S., Silverman, J., Thomas, C. M., Trust, T. J., Warn, P., and Rex, J. H. (2016) Alternatives to antibiotics-a pipeline portfolio review. Lancet Infect Dis 16, 239-251

3. Heselpoth, R. D., Swift, S.M., Linden, S.B., Mitchell, M.S., Nelson, D.C. (2018) Enzybiotics: Endolysins and bacteriocins. In Bacteriophages (Harper, D. R., Abedon, S. T., Burrowes, B. H., and McConville, M. L., eds), Springer International Publishing AG

4. Schmelcher, M., and Loessner, M. J. (2021) Bacteriophage endolysins - extending their application to tissues and the bloodstream. Curr Opin Biotechnol 68, 51-59

5. Danis-Wlodarczyk, K. M., Wozniak, D. J., and Abedon, S. T. (2021) Treating bacterial infections with bacteriophage-based enzybiotics: In vitro, in vivo and clinical application. Antibiotics (Basel) 10

6. Ho, M. K. Y., Zhang, P., Chen, X., Xia, J., and Leung, S. S. Y. (2021) Bacteriophage endolysins against gram-positive bacteria, an overview on the clinical development and recent advances on the delivery and formulation strategies. Crit Rev Microbiol, 1-24

7. Nelson, D., Loomis, L., and Fischetti, V. A. (2001) Prevention and elimination of upper respiratory colonization of mice by group A streptococci by using a bacteriophage lytic enzyme. Proceedings of the National Academy of Sciences of the United States of America 98, 4107-4112 
8. Linden, S. B., Alreja, A. B., and Nelson, D. C. (2021) Application of bacteriophage-derived endolysins to combat streptococcal disease: current state and perspectives. Curr Opin Biotechnol 68, 213-220

9. Shen, Y., Barros, M., Vennemann, T., Gallagher, D. T., Yin, Y., Linden, S. B., Heselpoth, R. D., Spencer, D. J., Donovan, D. M., Moult, J., Fischetti, V. A., Heinrich, F., Losche, M., and Nelson, D. C. (2016) A bacteriophage endolysin that eliminates intracellular streptococci. Elife 5

10. Shen, Y., Koller, T., Kreikemeyer, B., and Nelson, D. C. (2013) Rapid degradation of Streptococcus pyogenes biofilms by PlyC, a bacteriophage-encoded endolysin. $J$ Antimicrob Chemother 68, 1818-1824

11. McGowan, S., Buckle, A. M., Mitchell, M. S., Hoopes, J. T., Gallagher, D. T., Heselpoth, R. D., Shen, Y., Reboul, C. F., Law, R. H., Fischetti, V. A., Whisstock, J. C., and Nelson, D. C. (2012) X-ray crystal structure of the streptococcal specific phage lysin PlyC. Proceedings of the National Academy of Sciences of the United States of America 109, $12752-12757$

12. Broendum, S. S., Williams, D. E., Hayes, B. K., Kraus, F., Fodor, J., Clifton, B. E., Geert Volbeda, A., Codee, J. D. C., Riley, B. T., Drinkwater, N., Farrow, K. A., Tsyganov, K., Heselpoth, R. D., Nelson, D. C., Jackson, C. J., Buckle, A. M., and McGowan, S. (2021) High avidity drives the interaction between the streptococcal $\mathrm{C} 1$ phage endolysin, PlyC, with the cell surface carbohydrates of Group A Streptococcus. Mol Microbiol 116, 397-415

13. King, H., Ajay Castro, S., Pohane, A. A., Scholte, C. M., Fischetti, V. A., Korotkova, N., Nelson, D. C., and Dorfmueller, H. C. (2021) Molecular basis for recognition of the Group 
A Carbohydrate backbone by the PlyC streptococcal bacteriophage endolysin. Biochem J 478, 2385-2397

14. Anderson, G., and Scott, M. (1991) Determination of product shelf life and activation energy for five drugs of abuse. Clin Chem 37, 398-402

15. Blanco, I., Abate, L., and Antonelli, M. L. (2011) The regression of isothermal thermogravimetric data to evaluate degradation $E_{a}$ values of polymers: A comparison with literature methods and an evaluation of lifetime prediction reliability. Polym Degrad Stab 96, 1947-1954

16. Nelson, D., Schuch, R., Zhu, S., Tscherne, D. M., and Fischetti, V. A. (2003) Genomic sequence of $\mathrm{C} 1$, the first streptococcal phage. J Bacteriol 185, 3325-3332

17. Kirchhoff, W. H. (1993) Exam: A two-state thermodynamic analysis program. NIST Tech. Note 1401, 1-103

18. Manly, S. P., Matthews, K. S., and Sturtevant, J. M. (1985) Thermal denaturation of the core protein of lac repressor. Biochemistry 24, 3842-3846

19. Fischetti, V. A., Gotschlich, E. C., and Bernheimer, A. W. (1971) Purification and physical properties of group C streptococcal phage-associated lysin. J Exp Med 133, 1105-1117

20. Heselpoth, R. D., Yin, Y., Moult, J., and Nelson, D. C. (2015) Increasing the stability of the bacteriophage endolysin PlyC using rationale-based FoldX computational modeling. Protein Eng Des Sel 28, 85-92

21. Heselpoth, R. D., and Nelson, D. C. (2012) A new screening method for the directed evolution of thermostable bacteriolytic enzymes. J Vis Exp 
22. Kim, K. S., Kim, S., Yang, H. J., and Kwon, D. Y. (2004) Changes of glycinin conformation due to $\mathrm{pH}$, heat and salt determined by differential scanning calorimetry and circular dichroism. Int J Food Sci Tech 39, 385-393

23. Yamasaki, M., Yano, H., and Aoki, K. (1990) Differential scanning calorimetric studies on bovine serum albumin: I. Effects of $\mathrm{pH}$ and ionic strength. Int J Biol Macromol 12, 263268

24. Richard, A. J., Liu, C. C., Klinger, A. L., Todd, M. J., Mezzasalma, T. M., and LiCata, V. J. (2006) Thermal stability landscape for Klenow DNA polymerase as a function of $\mathrm{pH}$ and salt concentration. Biochim Biophys Acta 1764, 1546-1552

25. Bhat, R., and Timasheff, S. N. (1992) Steric Exclusion Is the Principal Source of the Preferential Hydration of Proteins in the Presence of Polyethylene Glycols. Protein Science 1, 1133-1143

26. Nelson, D., Schuch, R., Chahales, P., Zhu, S., and Fischetti, V. A. (2006) PlyC: a multimeric bacteriophage lysin. Proceedings of the National Academy of Sciences of the United States of America 103, 10765-10770

27. Kumar, S., Tsai, C. J., and Nussinov, R. (2000) Factors enhancing protein thermostability. Protein Eng 13, 179-191

28. Niu, C., Zhu, L., Xu, X., and Li, Q. (2016) Rational design of disulfide bonds increases thermostability of a mesophilic 1,3-1,4-beta-glucanase from Bacillus terquilensis. PLoS One 11, e 0154036

29. Liu, L., Deng, Z., Yang, H., Li, J., Shin, H. D., Chen, R. R., Du, G., and Chen, J. (2014) In silico rational design and systems engineering of disulfide bridges in the catalytic domain 
bioRxiv preprint doi: https://doi.org/10.1101/2022.01.06.475266; this version posted January 7, 2022. The copyright holder for this preprint

(which was not certified by peer review) is the author/funder. This article is a US Government work. It is not subject to copyright under 17 USC 105 and is also made available for use under a CCO license.

of an alkaline alpha-amylase from Alkalimonas amylolytica to improve thermostability.

Appl Environ Microbiol 80, 798-807 


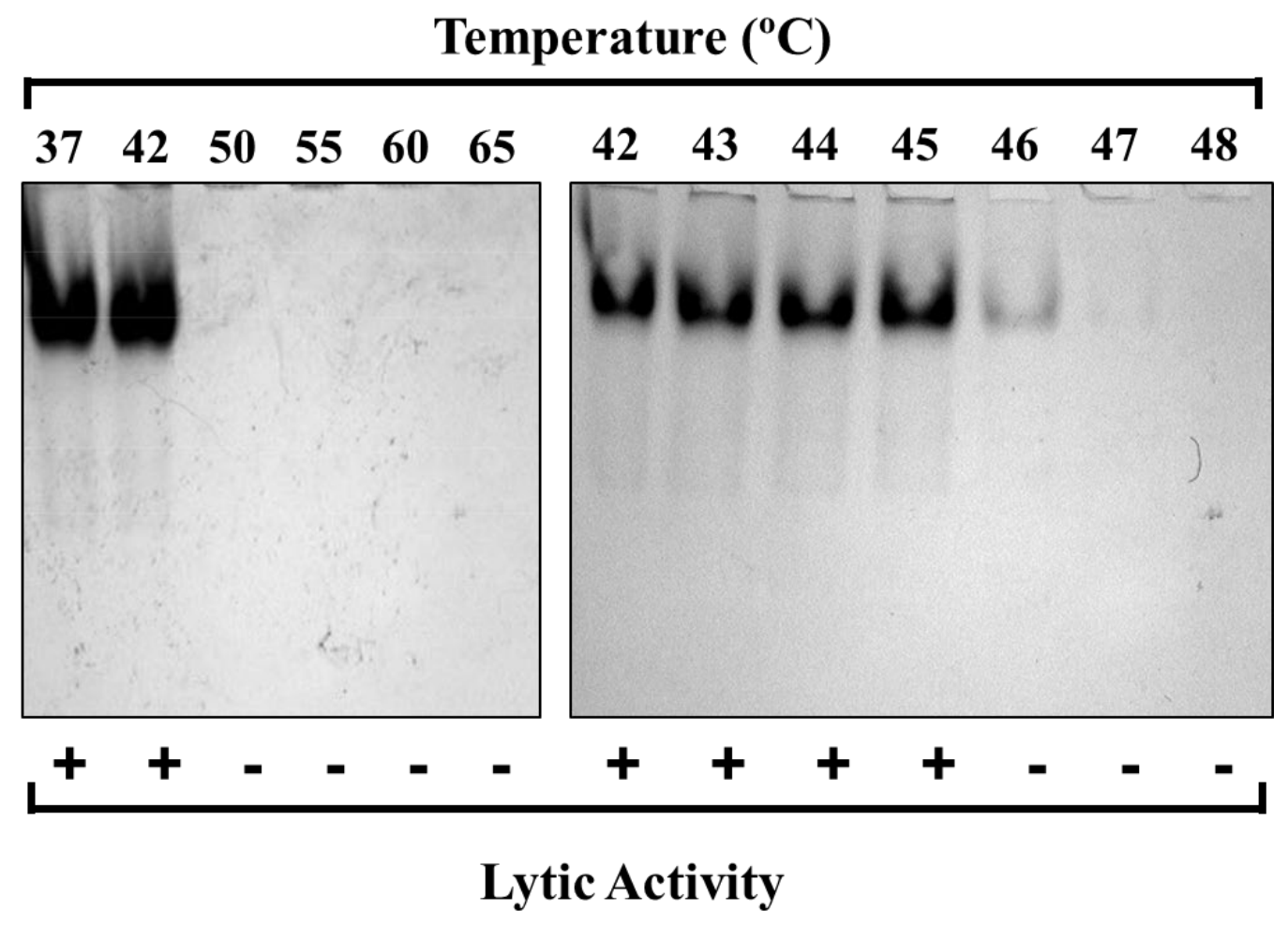

Figure 1. Thermal denaturation of PlyC. Top: The structural stability of the PlyC holoenzyme was assessed by incubating the endolysin at $1 \mathrm{mg} / \mathrm{ml}$ in PBS at temperatures ranging from 37 to $65^{\circ} \mathrm{C}$ (left) o 42 to $48^{\circ} \mathrm{C}$ (right) for $30 \mathrm{~min}$. After heat treatment, the samples were cooled on ice and visualized via native PAGE. Absence of PlyC in wells corresponds to thermally-induced protein unfolding and aggregation. Bottom: Lytic activity was normalized to unheated PlyC.,$+ \geq$ $90 \%$ of unheated PlyC activity;,$- \leq 10 \%$ of unheated PlyC activity. 


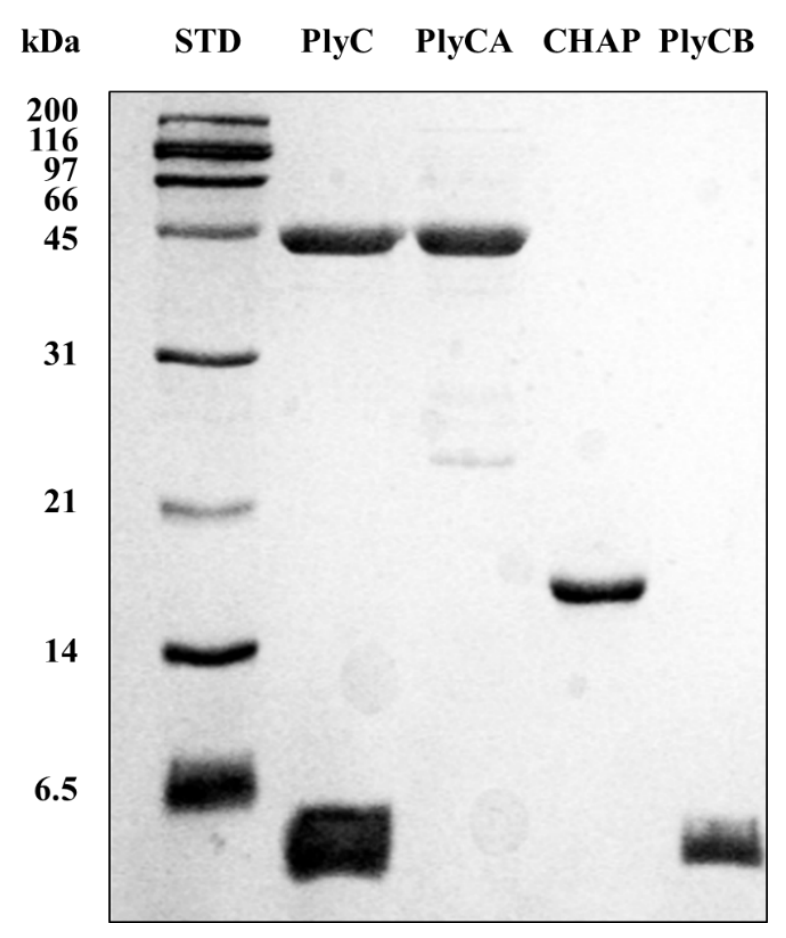

Figure 2. Purification of various PlyC-related constructs used in this study. The PlyC holoenzyme $(114 \mathrm{kDa}$; PlyCA subunit $=50 \mathrm{kDa}$, PlyCB subunit $=8 \mathrm{kDa})$, the PlyCA catalytic subunit $(50 \mathrm{kDa})$, the C-terminal CHAP domain of PlyCA $(18 \mathrm{kDa})$, and the PlyCB CBD (64 kDa; PlyCB subunit $=8 \mathrm{kDa}$ ) were purified to near homogeneity as indicated by SDS-PAGE. 
$\mathbf{A}$
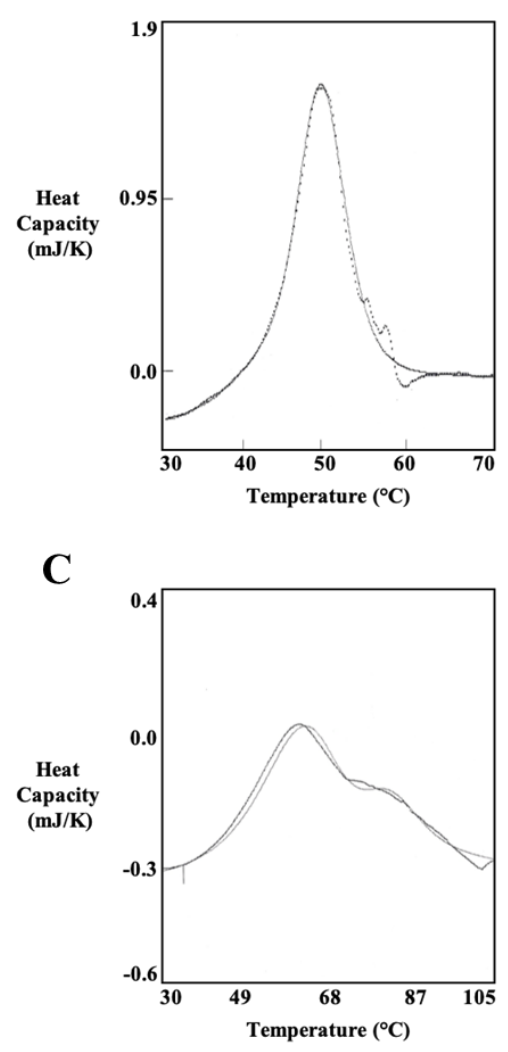

B
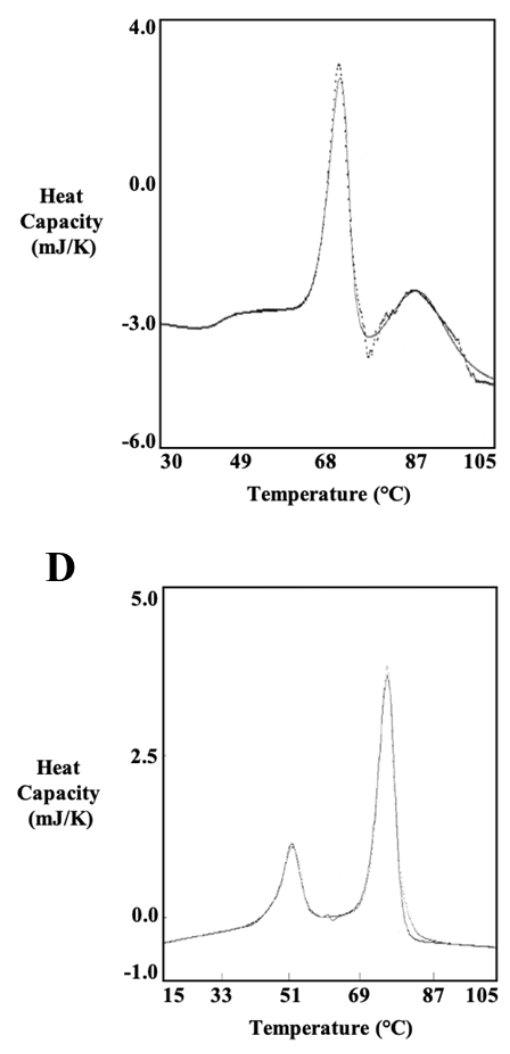

Figure 3. Thermodynamic analysis of PlyCA, PlyCB CBD and the PlyC holoenzyme using DSC. The thermodynamic properties of (A) PlyCA, (B) PlyCB CBD, (C) PlyCB CBD (rescanned) and (D) PlyC were experimentally determined using DSC. Each sample was heated in the calorimeter from 15 to $105^{\circ} \mathrm{C}$ and subsequently cooled from 105 to $15^{\circ} \mathrm{C}$ using scan rates of 15 or $60 \mathrm{~K} / \mathrm{h}$. The representative thermograms depicted were obtained from analyzing the protein samples in either PBS (PlyCA and PlyC) or phosphate buffer (PlyCB CBD). 

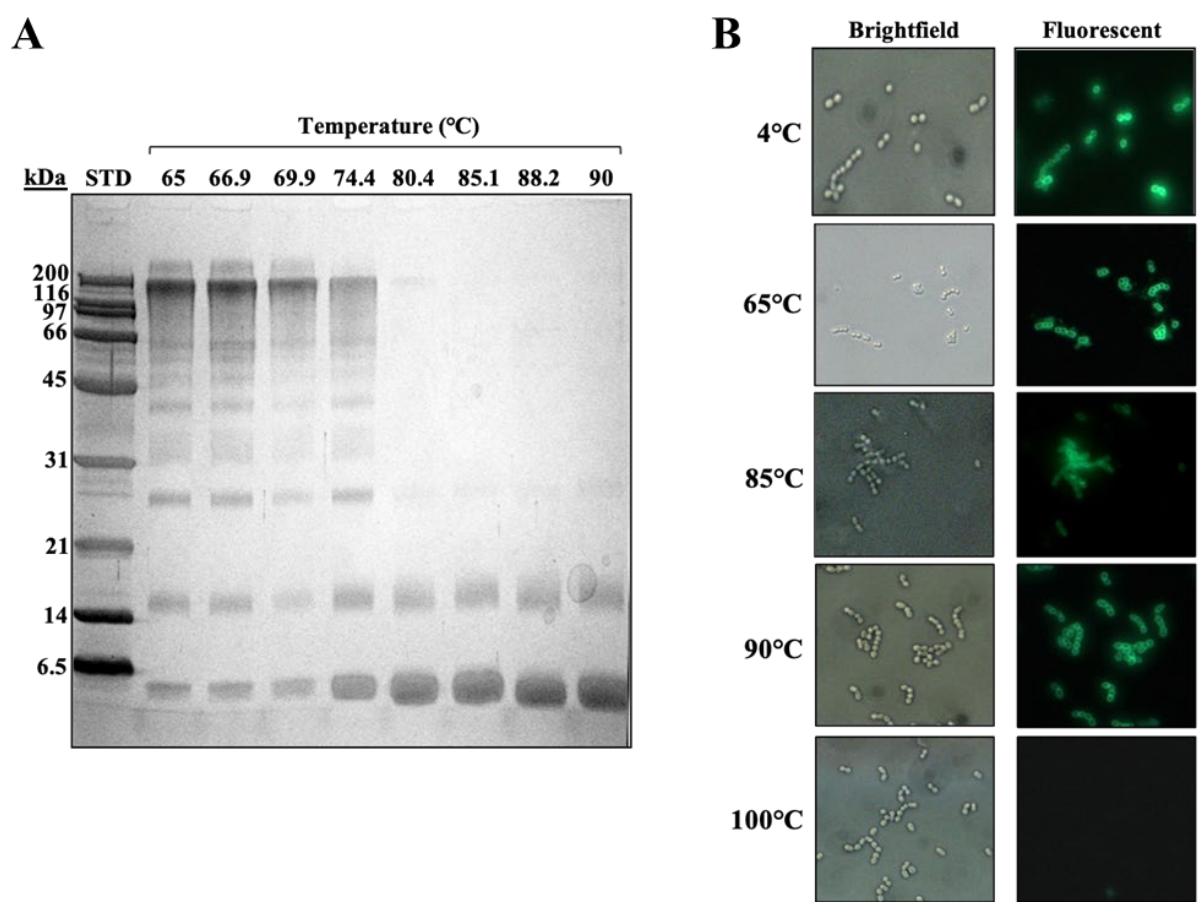

Figure 4. Structural and kinetic stability of the PlyCB octamer. (A) Following a 10-min incubation in PBS at temperatures ranging from 65 to $90^{\circ} \mathrm{C}$, PlyCB at $1 \mathrm{mg} / \mathrm{ml}$ was chemically cross-linked and cooled on ice. The samples were analyzed using SDS-PAGE in order to evaluate the structural integrity of the PlyCB octamer. (B) AlexaFluor-labeled PlyCB CBD at $1 \mathrm{mg} / \mathrm{ml}$ in PBS was incubated at 4 to $100^{\circ} \mathrm{C}$ for $30 \mathrm{~min}$, cooled on ice, and then added to $S$. pyogenes. The kinetic stability of the heat-treated PlyCB samples (i.e., PlyCB that retains its ability to bind $S$. pyogenes following heat treatment) was analyzed by means of fluorescence microscopy. 


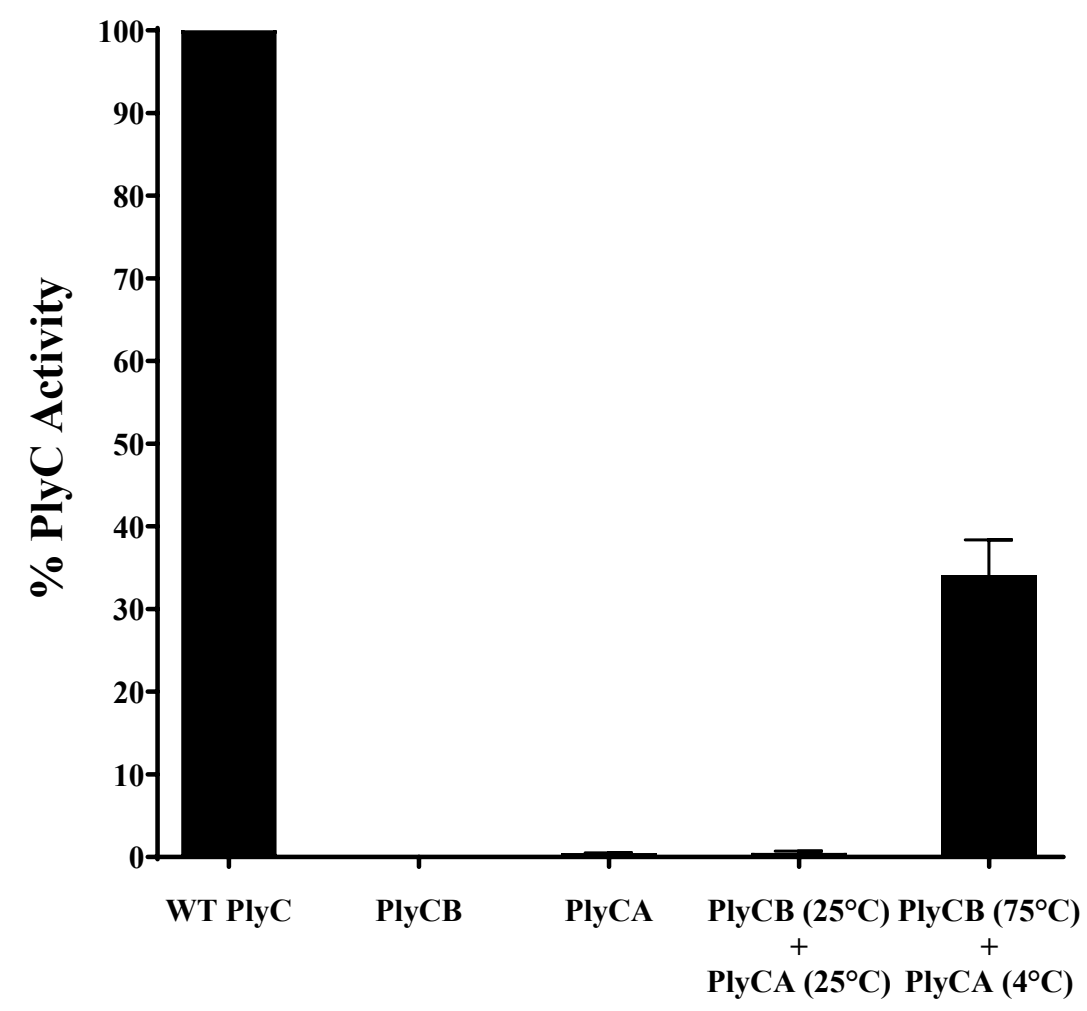

Figure 5. Thermal reconstitution of the PlyC holoenzyme. To elucidate the folding dynamics of PlyC, an attempt was made to reassemble the holoenzyme structure using purified PlyCA and PlyCB subunits. In one scenario, PlyCA and the PlyCB CBD were incubated at room temperature. Alternatively, ice-cold PlyCA was mixed with the PlyCB CBD in PBS heated to $75^{\circ} \mathrm{C}$ and then immediately placed on ice. A 1:1 molar ratio of PlyCA:PlyCB CBD was used in both instances. Used as an indirect measure of PlyC holoenzyme reconstitution, the bacteriolytic activity of each sample at equal molar concentrations was assayed against $S$. pyogenes using a turbidity reduction assay. Activity was normalized to that of PlyC. Isolated PlyCA $(<1 \%$ activity of PlyC) and PlyCB CBD (no activity) were used as controls. 
Table 1. Thermodynamic characterization of PlyCA in PBS by means of DSC.

\begin{tabular}{lllllll}
\hline $\begin{array}{l}\text { Conc. } \\
(\mathrm{mg} / \mathrm{ml})\end{array}$ & $\begin{array}{l}\text { Scan Rate } \\
(\mathrm{K} / \mathrm{h})\end{array}$ & Model & $T_{G}\left({ }^{\circ} \mathrm{C}\right)$ & $\begin{array}{l}\Delta H_{\mathrm{vH}} \\
(\mathrm{kJ} / \mathrm{mol})\end{array}$ & $\begin{array}{l}\Delta H_{\text {cal }} \\
(\mathrm{kJ} / \mathrm{mol})\end{array}$ & Ratio $^{\mathrm{a}}$ \\
\hline $1.0-2.0$ & 15 & $\mathrm{~A} \leftrightarrow \mathrm{B}$ & $41.4 \pm 1.7$ & $845 \pm 154$ & $1633 \pm 163$ & $1.08 \pm 0.25$ \\
& & $\mathrm{~A} \leftrightarrow \mathrm{B}$ & $44.6 \pm 0.5$ & $674 \pm 194$ & & \\
$1.0-2.0$ & 60 & $\mathrm{~A} \leftrightarrow \mathrm{B}$ & $42.0 \pm 2.3$ & $302 \pm 76$ & $922 \pm 205$ & $1.09 \pm 0.26$ \\
& & $\mathrm{~A} \leftrightarrow \mathrm{B}$ & $48.6 \pm 0.2$ & $545 \pm 81$ & & \\
\hline
\end{tabular}

${ }^{\mathrm{a}}$ Ratio equates to $\left[\Delta H_{\mathrm{cal}} /\left(\Delta H_{\mathrm{vH}}\right.\right.$ (low temperature $)+\Delta H_{\mathrm{vH}}$ (high temperature $\left.\left.)\right)\right]$ 
Table 2. Thermodynamic characterization of the CHAP domain in PBS by means of DSC.

\begin{tabular}{|c|c|c|c|c|c|c|}
\hline $\begin{array}{l}\text { Conc. } \\
(\mathrm{mg} / \mathrm{ml})\end{array}$ & $\begin{array}{l}\text { Scan Rate } \\
(\mathrm{K} / \mathrm{h})\end{array}$ & Model & $T_{G}\left({ }^{\circ} \mathrm{C}\right)$ & $\begin{array}{l}\Delta H_{\mathrm{vH}} \\
(\mathrm{kJ} / \mathrm{mol})\end{array}$ & $\begin{array}{l}\Delta H_{\mathrm{cal}} \\
(\mathrm{kJ} / \mathrm{mol})\end{array}$ & Ratio $^{a}$ \\
\hline \multirow[t]{2}{*}{1.0} & 15 & $\mathrm{~A} \leftrightarrow \mathrm{B}$ & $38.5 \pm 0.5$ & $464 \pm 2$ & $439 \pm 44$ & $0.42 \pm 0.04$ \\
\hline & & $\mathrm{A} \leftrightarrow \mathrm{B}$ & $44.3 \pm 0.5$ & $583 \pm 58$ & & \\
\hline \multirow[t]{2}{*}{$1.0-2.0$} & 60 & $\mathrm{~A} \leftrightarrow \mathrm{B}$ & $39.1 \pm 0.4$ & $430 \pm 70$ & $394 \pm 3$ & $0.40 \pm 0.07$ \\
\hline & & $\mathrm{A} \leftrightarrow \mathrm{B}$ & $46.1 \pm 0.6$ & $554 \pm 6$ & & \\
\hline
\end{tabular}


Table 3. Thermodynamic characterization of the PlyCB CBD in PBS by means of DSC.

\begin{tabular}{llllllll}
\hline & $\begin{array}{l}\text { Conc. } \\
(\mathrm{mg} / \mathrm{ml})\end{array}$ & $\begin{array}{l}\text { Scan Rate } \\
(\mathrm{K} / \mathrm{h})\end{array}$ & Model & $T_{G}\left({ }^{\circ} \mathrm{C}\right)$ & $\begin{array}{l}\Delta H_{\mathrm{vH}} \\
(\mathrm{kJ} / \mathrm{mol})\end{array}$ & $\begin{array}{l}\Delta H_{\mathrm{cal}} \\
(\mathrm{kJ} / \mathrm{mol})\end{array}$ & Ratio $^{\mathrm{a}}$ \\
\hline & 1.0 & 60 & $\mathrm{~A}_{2} \leftrightarrow 2 \mathrm{~B}$ & $73.7 \pm 0.5$ & $1265 \pm 53$ & $2371 \pm 237$ & $1.87 \pm 0.21$ \\
& & $\mathrm{~A} \leftrightarrow \mathrm{B}$ & $91.8 \pm 0.1$ & $368 \pm 37$ & \\
$\begin{array}{l}\text { Rescan to } \\
95^{\circ} \mathrm{C}\end{array}$ & 1.0 & 60 & $\mathrm{~A}_{2} \leftrightarrow 2 \mathrm{~B}$ & $49.4 \pm 0.1$ & $275 \pm 4$ & $93 \pm 10$ \\
& & $\mathrm{~A} \leftrightarrow \mathrm{B}$ & $67.0 \pm 0.1$ & $230 \pm 23$ & $275 \pm 28$ \\
$\begin{array}{l}\text { Rescan to } \\
105^{\circ} \mathrm{C}\end{array}$ & 1.0 & 60 & $\mathrm{~A}_{2} \leftrightarrow 2 \mathrm{~B}$ & $68.2 \pm 0.1$ & $263 \pm 26$ & $148 \pm 15$ \\
& & $\mathrm{~A} \leftrightarrow \mathrm{B}$ & $91.5 \pm 0.1$ & $650 \pm 65$ & $57 \pm 6$ \\
\hline
\end{tabular}

${ }^{\text {aRatio equates to }\left[\Delta H_{\mathrm{cal}} /\left(\Delta H_{\mathrm{vH}}(\text { low temperature })\right)\right]}$ 
Table 4. Thermodynamic characterization of the PlyCB CBD in phosphate buffer by means of DSC.

\begin{tabular}{llllllll}
\hline & $\begin{array}{l}\text { Conc. } \\
(\mathrm{mg} / \mathrm{ml})\end{array}$ & $\begin{array}{l}\text { Scan Rate } \\
(\mathrm{K} / \mathrm{h})\end{array}$ & Model & $T_{G}\left({ }^{\circ} \mathrm{C}\right)$ & $\begin{array}{l}\Delta H_{\mathrm{vH}} \\
(\mathrm{kJ} / \mathrm{mol})\end{array}$ & $\begin{array}{l}\Delta H_{\mathrm{cal}} \\
(\mathrm{kJ} / \mathrm{mol})\end{array}$ & Ratio $^{\mathrm{a}}$ \\
\hline & $1.0-2.0$ & 60 & $\mathrm{~A}_{2} \leftrightarrow 2 \mathrm{~B}$ & $70.4 \pm 1.4$ & $1160 \pm 119$ & $2349 \pm 235$ & $2.03 \pm 0.28$ \\
& & $\mathrm{~A} \leftrightarrow \mathrm{B}$ & $83.5 \pm 2.7$ & $373 \pm 84$ & \\
$\begin{array}{l}\text { Rescan to } \\
75^{\circ} \mathrm{C}\end{array}$ & 1.0 & 60 & $\mathrm{~A}_{2} \leftrightarrow 2 \mathrm{~B}$ & $68.8 \pm 0.1$ & $945 \pm 95$ & $1870 \pm 187$ \\
$\begin{array}{l}\text { Rescan to } \\
95^{\circ} \mathrm{C}\end{array}$ & 2.0 & 60 & $\mathrm{~A}_{2} \leftrightarrow 2 \mathrm{~B}$ & $63.0 \pm 0.1$ & $427 \pm 43$ & $281 \pm 28$ \\
& & & $\mathrm{~A} \leftrightarrow \mathrm{B}$ & $82.4 \pm 0.1$ & $405 \pm 13$ & \\
$\begin{array}{l}\text { Rescan to } \\
105^{\circ} \mathrm{C}\end{array}$ & 1.0 & 60 & $\mathrm{~A} 2 \leftrightarrow 2 \mathrm{~B}$ & $58.1 \pm 0.1$ & $241 \pm 24$ & $555 \pm 56$ \\
& & $\mathrm{~A} \leftrightarrow \mathrm{B}$ & $82.1 \pm 0.1$ & $227 \pm 3$ & \\
\hline
\end{tabular}

${ }^{\text {a Ratio equates to }\left[\Delta H_{\mathrm{cal}} /\left(\Delta H_{\mathrm{vH}}(\text { low temperature })\right)\right]}$ 
Table 5. Thermodynamic characterization of PlyC in PBS by means of DSC.

\begin{tabular}{|c|c|c|c|c|c|c|c|}
\hline & $\begin{array}{l}\text { Conc. } \\
(\mathrm{mg} / \mathrm{ml})\end{array}$ & $\begin{array}{l}\text { Scan Rate } \\
(\mathrm{K} / \mathrm{h})\end{array}$ & Model & $T_{G}\left({ }^{\circ} \mathrm{C}\right)$ & $\begin{array}{l}\Delta H_{\mathrm{vH}} \\
(\mathrm{kJ} / \mathrm{mol})\end{array}$ & $\begin{array}{l}\Delta H_{\mathrm{cal}} \\
(\mathrm{kJ} / \mathrm{mol})\end{array}$ & Ratio $^{a}$ \\
\hline & \multirow[t]{3}{*}{$0.81-3.82$} & \multirow[t]{3}{*}{15} & $\mathrm{~A} \leftrightarrow \mathrm{B}$ & $44.5 \pm 0.5$ & $677 \pm 42$ & $501 \pm 72$ & $0.33 \pm 0.04$ \\
\hline & & & $\mathrm{A} \leftrightarrow \mathrm{B}$ & $48.4 \pm 0.2$ & $862 \pm 105$ & & \\
\hline & & & $\mathrm{A}_{2} \leftrightarrow 2 \mathrm{~B}$ & $73.7 \pm 0.4$ & $1238 \pm 76$ & $1857 \pm 979$ & $1.5 \pm 0.6$ \\
\hline & \multirow[t]{3}{*}{$1.0-2.25$} & \multirow[t]{3}{*}{60} & $\mathrm{~A} \leftrightarrow \mathrm{B}$ & $46.2 \pm 1.3$ & $472 \pm 92$ & $637 \pm 41$ & $0.57 \pm 0.11$ \\
\hline & & & $\mathrm{A} \leftrightarrow \mathrm{B}$ & $50.6 \pm 0.5$ & $637 \pm 41$ & & \\
\hline & & & $\mathrm{A}_{2} \leftrightarrow 2 \mathrm{~B}$ & $75.0 \pm 0.1$ & $1082 \pm 33$ & $2075 \pm 168$ & $1.92 \pm 0.17$ \\
\hline $\begin{array}{l}\text { Rescan to } \\
95^{\circ} \mathrm{C}\end{array}$ & $1.0-2.25$ & 60 & $\mathrm{~A}_{2} \leftrightarrow 2 \mathrm{~B}$ & $68.9 \pm 1.8$ & $486 \pm 149$ & $143 \pm 12$ & \\
\hline $\begin{array}{l}\text { Rescan to } \\
105^{\circ} \mathrm{C}\end{array}$ & $1.0-2.25$ & 60 & $\mathrm{~A}_{2} \leftrightarrow 2 \mathrm{~B}$ & $68.3 \pm 0.5$ & $430 \pm 10$ & $62 \pm 6$ & \\
\hline
\end{tabular}

${ }^{\mathrm{a}}$ Ratio equates to $\left[\Delta H_{\mathrm{cal}} /\left(\Delta H_{\mathrm{vH}}(\right.\right.$ low temperature $\left.\left.)\right)\right]$ 